\title{
EDITORIAL
}

\section{Dissemination of scientific knowledge and information}

Science and technology are two of the most important drivers that have influenced individual, societal, national and global changes and development. It is therefore necessary for all to know and understand the scientific principles underlying the natural processes as well as the causes and consequences, both beneficial and adverse, of scientific developments and innovations. These have deep ethical, legal and social implications. Therefore, it is essential that scientific knowledge and information is disseminated to all levels of society.

It is noteworthy that the Government of Sri Lanka has emphasised the importance of dissemination of scientific knowledge in several policy documents. The Parliament-approved Science and Technology (S\&T) Act No. 11 of 1994, the National Science and Technology Policy 2009 and the Science, Technology and Innovation Strategy 2011 - 2015 have all incorporated measures regarding the dissemination of scientific knowledge and information among various categories of persons in the country.

The S\&T Act lists, among its objectives, "the provision of adequate opportunities for all persons to acquire a basic education in science and its applications and the cultivation of appreciation of the value of science, the scientific method and technology". The National S\&T Policy, which has ten objectives, lists as the first "foster a science, technology and innovation culture that efficiently reaches all citizens of the country". It has also proposed as an 'initiative', popularising science through targeted activities by S\&T institutions and professional associations. One of the goals of the Science, Technology and Innovation Strategy is "An effective framework to prepare the people of Sri Lanka for a knowledge society". Under this goal is listed the objective of implementing the "science for all initiative", which will require the efficient and satisfactory dissemination of scientific knowledge and information.

The S\&T Act has assigned the function of popularising science amongst people of the country and the fostering of the interchange of scientific information amongst scientists in Sri Lanka and foreign countries to the National Science Foundation (NSF). The National Science Library and Resource Centre (NSLRC) of the NSF is the focal point for dissemination of S\&T information in the country. To ensure that the message of science reaches most citizens in Sri Lanka, the NSF has developed various initiatives.

These initiatives include the Sri Lanka Science and Technology Information Network (SLSTINET), which is operated among over 100 S\&T libraries. The website of the NSLRC functions as the central hub linking S\&T related information sources for efficient exchange of information. The NSLRC has an extensive collection of S\&T related books, which are accessible online. In addition to the above, the NSLRC houses the national collection of scholarly scientific literature that includes local and foreign publications in journals as well as conference presentations. Also available are unpublished research reports, technical reports, theses and conference papers. These are all available online. The investments made on providing these facilities will become cost-effective when a large number of scientists and others make use of them.

The NSF publishes two research journals, the Journal of the National Science Foundation of Sri Lanka (JNSF) and the Sri Lanka Journal of Social Sciences (SLJSS). In addition, NSF publishes a monograph series, reports of NSF funded research and Vidurawa, a popular science magazine. The maintenance of a complete collection 
of locally published journals available in digital form for online access within the NSF premises is another initiative of the NSLRC.

Scientific information is disseminated by scientists among other scientists through research papers published in scientific journals. Writing to the scientific community is rather straightforward since research findings are presented on an agreed structural basis. On the other hand, the art of writing to the general public does not come easy to scientists even though it is increasingly accepted as a duty and a responsibility. The responsibility of disseminating scientific knowledge and research information also lies with the science educators at all levels, scientists and both print and electronic media.

Many of the faculties in universities in Sri Lanka publish journals to disseminate their research findings. The information in these journals reaches fellow scientists but not the public in most instances. The scientists also have the responsibility to reach out to the general public. This can be achieved through both print and electronic media, but it does not appear to happen adequately. This task can be effectively performed by science journalists teaming up with scientists. However, it is often difficult to find media-friendly scientists. This situation needs the attention of both scientists and media personnel.

It is necessary to find new and innovative ways of mass communication to disseminate scientific information to the public. Mass media is the major player that can stimulate public debate and shape public opinion on scientific issues that impact society. It is encouraging to note that many professional and scientific associations conduct regular activities to reach out to school children and the public. However, these activities are limited due to inadequate funding and other resources. The government and the corporate sector must consider this seriously and help the science communicators to further their activities.

Nalini Ratnasiri 